\title{
EL LAPBOOK COMO EXPERIENCIA EDUCATIVA
}

The lapbook as an educational experience

O lapbook como uma experiência educativa

\section{Laura Álvarez Cañas (1)}

Hugo Medina Melcón (2)

(1) Centro Rural Agrupado Ruta de la Plata, León, España. Teléfono: +34 676676655.

Correo electrónico: guasariiii@hotmail.com

(2) Centro Rural Agrupado Vega del Esla, León, España. Teléfono: +34 620540172

Correo electrónico: hugo.opositta@gmail.com

\section{Resumen}

La experiencia muestra cómo se puede aplicar el uso del lapbook en las aulas de Educación Primaria en los Colegios Rurales Agrupados. El lapbook, supone una alternativa a la pedagogía tradicional, es un libro interactivo en el cual el alumno muestra de una forma dinámica, activa y participativa los conocimientos que van adquiriendo a lo largo de las unidades didácticas, siendo partícipes de su propio proceso de enseñanza- aprendizaje a través de procesos de autorregulación y evaluación formativa permitiéndoles la adquisición de aprendizajes significativos. Para ello, tomando como modelo el lapbook elaborado por el profesor, los alumnos deben sintetizar toda aquella información que quieren que aparezca reflejada en el mismo.

Palabras clave: lapbook; aprendizajes significativo; evaluación formativa; autorregulación

\begin{abstract}
This experience shows how the use of the lapbook can be applied in the Primary Education classrooms in the Grouped Rural Schools. The lapbook, an alternative to traditional pedagogy, is an interactive book in which the student shows in a dynamic, active and participative way the knowledge they acquire throughout the didactic units, being participants in their own teaching process - learning through processes of selfregulation and formative evaluation allowing them to acquire meaningful learning. To
\end{abstract}


do this, taking as a model the lapbook prepared by the teacher, the students must synthesize all the information they want to appear reflected in it.

Keywords: Lapbook; meaningful learning; formative assessment; self-regulation

\section{Resumo}

A experiência mostra como você pode aplicar o uso lapbook nas salas de aula do ensino fundamental em escolas rurais agrupados. O lapbook, é uma alternativa à pedagogia tradicional, é um livro interativo no qual o aluno mostra um conhecimento mais dinâmica, ativa e participativa eles adquirem ao longo do formulário de unidades didácticas, sendo participantes em seu próprio processo de aprendizagem aprendizagem através de processos de auto-regulação e avaliação formativa permitindo a aquisição de aprendizagens significativas. Para isso, modelado no lapbook preparado pelo professor, os alunos devem sintetizar toda a informação que deseja que apareça refletido nele.

Palavras-chave: Lapbook; aprendizagens significativas; avaliação formativa; autoregulação

\section{Introducción}

La palabra inglesa "lapbook" significa libro con solapas. Tras la lectura de diversos blogs sobre materia educativa, se deduce que el uso del lapbook tiene su origen en la pedagogía del Homeschooling, es decir, la educación desde casa, movimiento creado en Estados Unidos en la década de 1970. Este movimiento pedagógico está basado en gran parte en la obra de Illich (1979), el cual pensaba que la mejor educación no tiene por qué impartirse desde una institución. Actualmente, innumerables páginas web de carácter educativo, así como aplicaciones como Pinterest, ofrecen múltiples ejemplos sobre cómo trabajar el lapbook en el aula, mostrando su gran potencial para la consecución de aprendizajes significativos (Ausubel, 1968).

En este caso, la experiencia se vincula a procesos de aprendizaje por autorregulación, el cual, se revela como una metodología de transición entre los modelos tradicionales de enseñanza-aprendizaje y aquellas propuestas basadas en el Aprendizaje Cooperativo o en el Aprendizaje Basado en Proyectos (Casado, PérezPueyo \& Casado, 2017). 


\section{Contextualización}

La experiencia ha sido llevada a cabo durante los cursos 2015-2016 y 2016-2017 en varias localidades pertenecientes al Centro Rural Agrupado "Ruta de la Plata”, en la provincia de León. Las aulas están formadas por alumnos de diferentes niveles, desde $1^{\circ}$ hasta $6^{\circ}$ de primaria, siendo Ciencias Sociales y Naturales las áreas escogidas para llevar a cabo el trabajo con el lapbook. Se llevó a cabo en dos aulas, una compuesta por cuatro alumnos (uno de $1^{\mathrm{o}}$, una de $2^{\circ} \mathrm{y}$ dos de $3^{\mathrm{er}}$ curso) y otra por 9 alumnos (uno de $1^{\mathrm{o}}$, tres de $4^{\circ}$ y tres de $6^{\circ}$ curso.)

\section{Diseño y desarrollo}

Para llevar a cabo el lapbook, lo primero que se debe tener claro es el contenido que se quiere abordar y después pensar sobre la forma en qué se va a presentar. Por ejemplo, si estamos trabajando las plantas, el lapbook puede tener forma de planta (Figura 1) o un cohete en el caso de estar abordando el sistema solar (Figura 2).

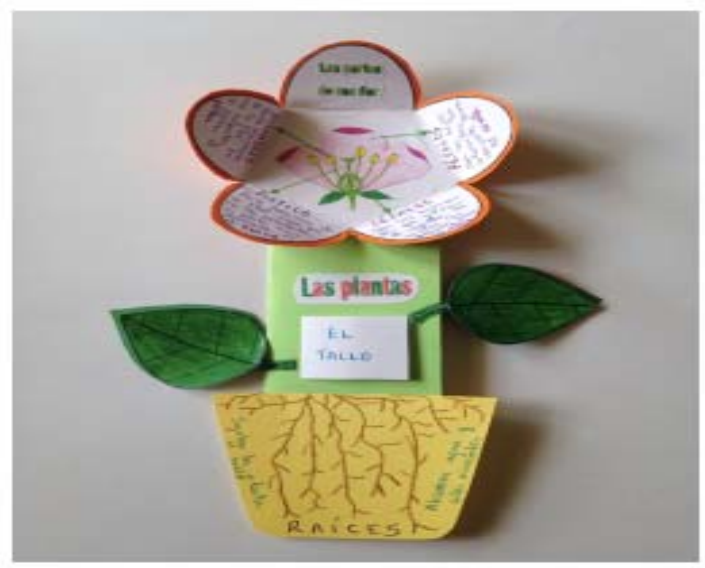

Figura 1.

Lapbook plantas (elaboración propia, 2016)

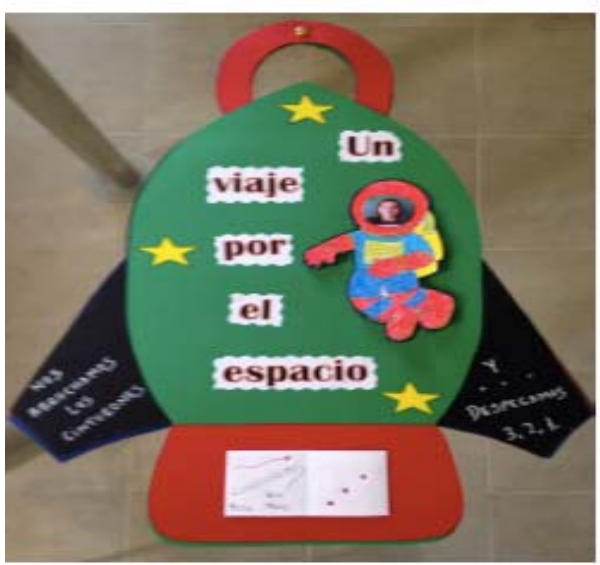

Figura 2

Lapbook espacio (elaboración propia, 2016)

Para poder realizarlo necesitamos diversos materiales: cartulinas, folios, dibujos, pinturas, pegamento, tijeras, plantillas, etc. El lapbook es un recurso útil y visual, permitiéndonos aprender y descubrir cosas nuevas interactuando con ellos.

La información que se quiera mostrar marcará el nivel de profundización con el que se realiza el lapbook pudiendo ser utilizado, entre otros, para diversos fines (Figura 3):

- Como actividad de evaluación, para comprobar el grado de adquisición de los contenidos tratados en la unidad didáctica. 
- Como actividad de síntesis final de la unidad.

- Como actividad de ampliación, planteado como un trabajo de investigación sobre aquellos aspectos que queramos incidir.

- Como actividad mixta que aglutine aspectos de las actividades anteriores, es decir, dentro del mismo trabajo se pueden hacer actividades de evaluación, síntesis final y de ampliación.

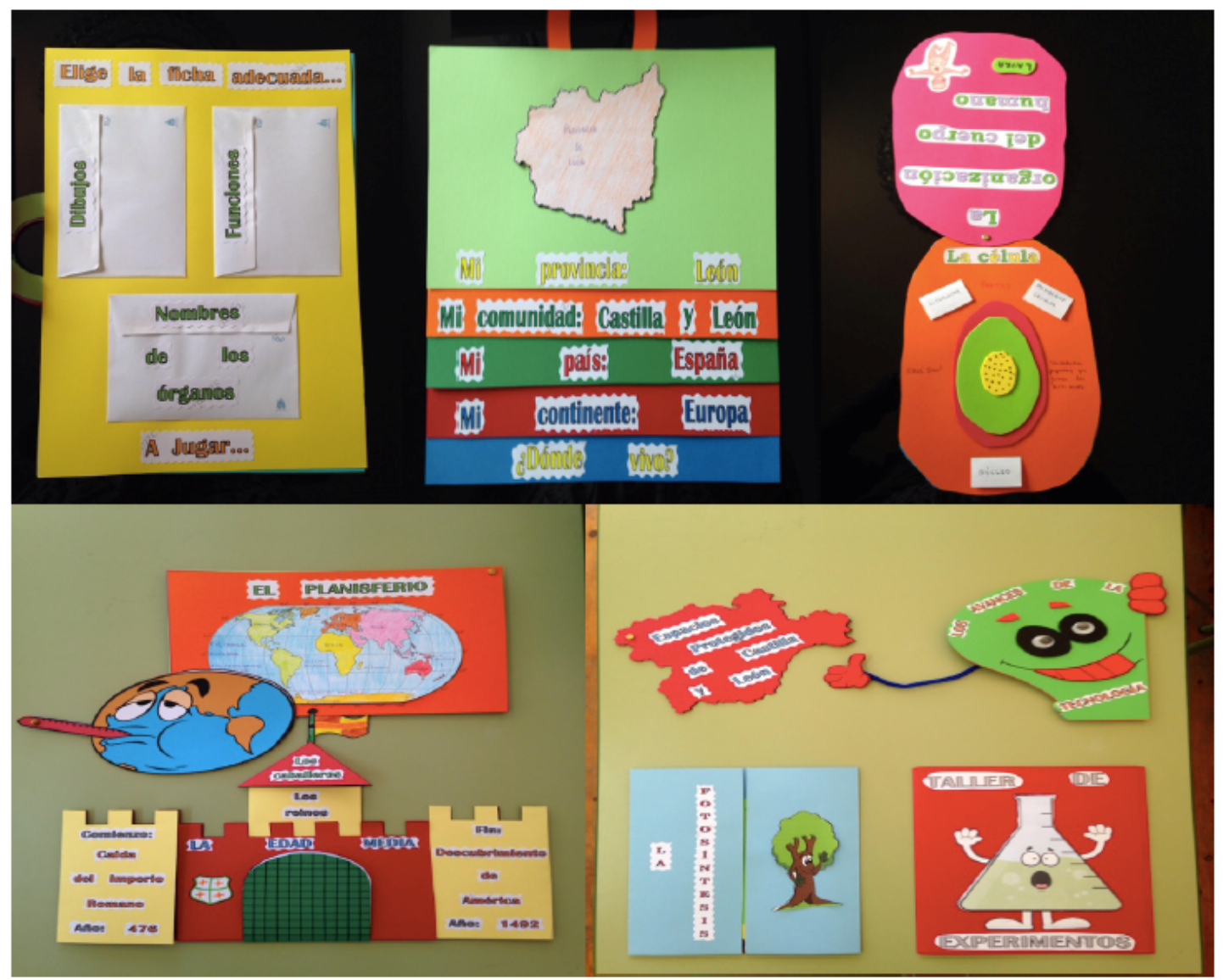

Figura 3.

Ejemplos de actividades de lapbook (elaboración propia, 2016,2017)

En función de la temática de las unidades didácticas, es el profesor quien estableció qué tipo de lapbook se iba a realizar, qué contenidos iban a aparecer reflejados en el mismo, así como las plantillas donde escribir toda la información pertinente. Para ello, elaboró, su propio lapbook como modelo, para posteriormente dar a los alumnos todo el material necesario para realizarlo, ya que requiere de una fase de planificación exhaustiva. Así, los alumnos iban realizando las actividades propuestas por el docente participando de forma activa en su desarrollo. 


\section{Evaluación}

A la hora de llevar a cabo la evaluación del lapbook, ésta va a depender de su finalidad. Generalmente, dentro de la unidad, se llevó a cabo su trabajo como una actividad de síntesis, ampliación y evaluación de los contenidos. Cobrando especial relevancia el concepto de evaluación formativa (López-Pastor, 2006) ya que, a través de la misma se pretendió mejorar y optimizar los procesos de enseñanza-aprendizaje.

Así, una vez realizado, los alumnos debían autoevaluar y autocalificar su trabajo en función de dos aspectos: a) La presentación: los márgenes, la claridad, la limpieza, el estilo y la originalidad; y b) El contenido: si todos los apartados aparecían correctamente. De este modo, el hecho de que participasen activamente en su propia evaluación implicaba una mayor responsabilidad por su parte, siendo conscientes de qué aspectos habían realizado bien y aquellos que debían resolver para poder mejorar sus aprendizajes.

A continuación se muestra un una rúbrica de autoevaluación para los alumnos de $5^{\circ}$ y $6^{\circ}$ modificada de Casado (2014) (Figura 4)

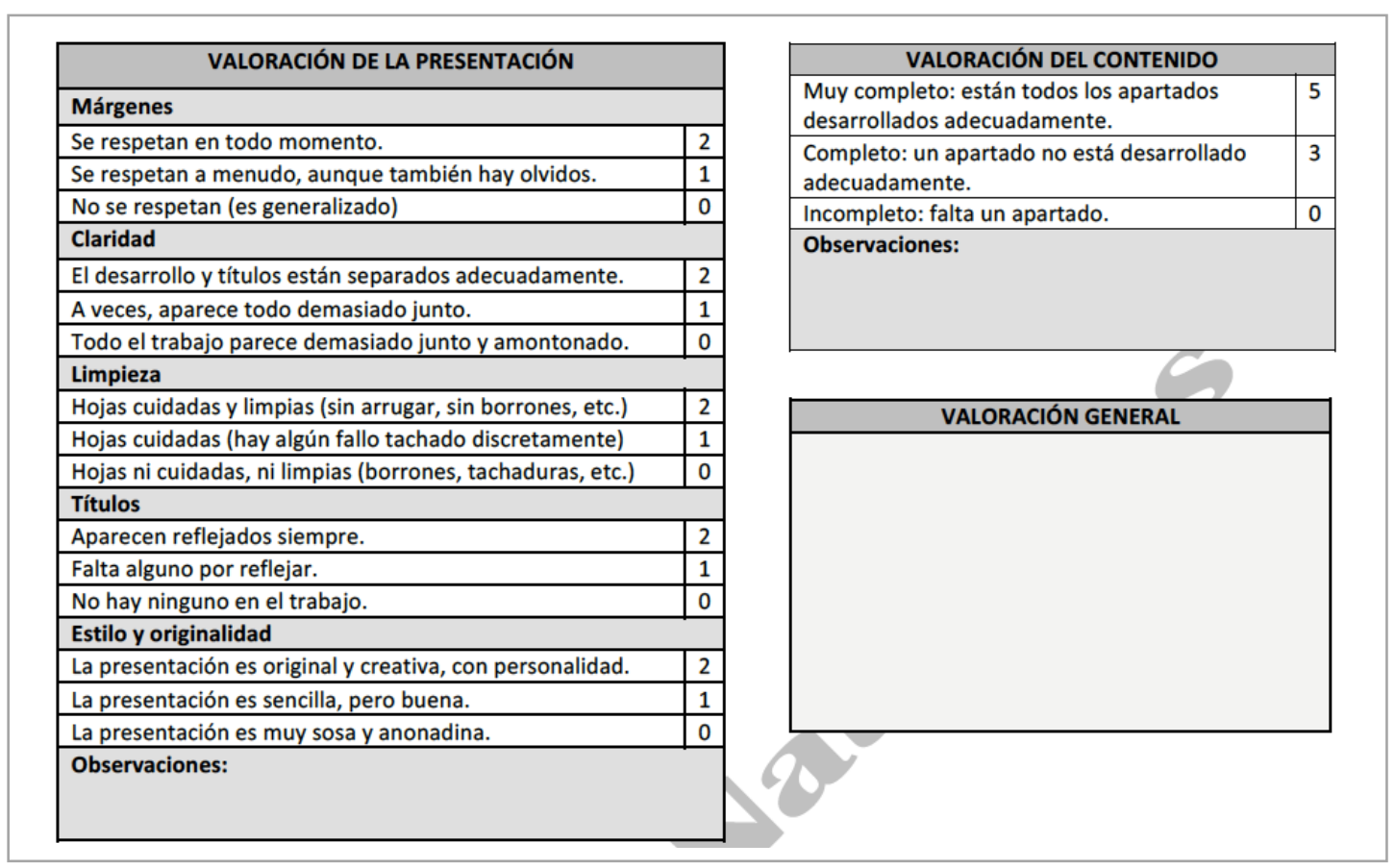

Figura 4.

Rúbrica de evaluación y calificación del lapbook (modificado de Casado, 2014)

La evaluación de la experiencia por parte de los alumnos, se llevó a cabo a través de un intercambio oral con ellos, donde comentaron lo mucho que les gustaba 
realizar este tipo de actividades ya que aprendían muchas más cosas. Como docente, lo más reseñable, es la motivación que tienen los alumnos al realizar este tipo de trabajos, ya que en innumerables situaciones, sin que ellos se den cuenta, es una forma indirecta de comprobar el aprendizaje adquirido sin la presión psicológica de preguntar la lección o de hacer un examen. Como van siendo partícipes de su proceso de aprendizaje y de forma espontánea se van afianzando las relaciones sociales al llevar a cabo la actividad de forma conjunta con sus compañeros.

\section{Conclusiones}

Al estar en un aula multinivelar, puede dar la sensación de que puede ser un poco caótico llevar a cabo estos trabajos, pero nada más lejos de la realidad. Lo único que requiere es de una buena planificación por parte del docente así como una actitud positiva, partiendo de la idea del “enfoque optimista” de López-Pastor (1999) con respecto a la educación en la escuela rural.

En este sentido, debemos adaptarnos a la realidad donde llevamos a cabo nuestra labor docente, aprovechando al máximo las posibilidades que nos ofrece el contexto y aceptar e intentar superar sus limitaciones pasando del "no puedo hacer porque..." al “que puedo hacer en...”; huyendo de las excusas y resolviendo los problemas como propone Pérez-Pueyo (2010).

\section{Referencias}

Ausubel, D. (1968). Procesos psicológicos básicos. Madrid: Laia.

Casado, O. (2014). Rúbrica para la valoración de los cuadernos de trabajo personal Recuperado de https://drive.google.com/file/d/0B7xEdPlO4dj8Z3VrSlZOQXdNYm8/view

Casado, O., Pérez-Pueyo, A., \& Casado, P. (2017). La autorregulación en educación primaria. Una propuesta para favorecer la autonomía del alumnado (pp. 208-237). En V. M. López-Pastor y A. Pérez-Pueyo (coords.) Evaluación formativa y compartida en Educación: experiencias de éxito en todas las etapas educativas. León: Universidad de León.

Illich, I. (1970). La sociedad desescolarizada. Barcelona: Barral Editores. 
López-Pastor, V. M. (Coord.). (1999). La Educación Física en la Escuela Rural. Buenos Aires: Miño y Dávila.

López-Pastor, V. M. (Coord.) (2006). La Evaluación en Educación Física: Revisión de los modelos tradicionales y planteamiento de una alternativa: La evaluación formativa y compartida. Buenos Aires: Miño y Dávila.

Pérez-Pueyo, A. (2010). El Estilo Actitudinal. Propuesta metodológica para desarrollar unidades didácticas en educación física. Madrid: CEP. 